\title{
Cingulate gyral reductions are related to low executive functioning and psychotic symptoms in 22q11.2 deletion syndrome
}

\author{
Federico Dufour ${ }^{\mathrm{a}, *}$, Marie Schaer ${ }^{\mathrm{a}, \mathrm{d}}$, Martin Debbané ${ }^{\mathrm{a}, \mathrm{b}}$, Riaz Farhoumand ${ }^{\mathrm{a}}$, \\ Bronwyn Glaser ${ }^{\mathrm{a}, \mathrm{b}}$, Stephan Eliez ${ }^{\mathrm{a}, \mathrm{c}}$ \\ a Service Médico-Pédagogique Research Unit, Department of Psychiatry, University of Geneva School of Medicine, 1 rue David-Dufour, Case Postale 50, 1211 Geneva 8, Switzerland \\ ${ }^{\mathrm{b}}$ Faculty of Psychology, University of Geneva, Switzerland \\ ${ }^{c}$ Department of Genetic Medicine and Development, University of Geneva School of Medicine, Switzerland \\ d Signal Processing Institute, Swiss Federal Institute of Technology, Lausanne, Switzerland
}

\section{A R T I C L E I N F O}

\section{Article history:}

Received 5 October 2007

Received in revised form 27 May 2008

Accepted 9 June 2008

Available online 21 June 2008

\section{Keywords:}

Cingulate gyrus

Neuroimaging

Psychosis

Velo-cardio-facial

Executive function

Schizophrenia

\begin{abstract}
A B S T R A C T
A similar pattern of deficits in executive function and neuroanatomical abnormalities is shared between 22q11.2 deletion syndrome (22q11DS) and schizophrenia, suggesting that common cerebral alterations may lead to cognitive dysfunction and promote the appearance of psychotic symptoms in 22q11DS individuals. Specifically, there is increasing evidence for involvement of the cingulate gyrus (CG) in executive dysfunction and the expression of positive symptoms in schizophrenia. The aim of our study is to examine CG morphology in a 22q11DS population and its potential role as a cerebral marker of executive dysfunction and the manifestation of psychotic symptoms. Using region of interest (ROI)-based analysis, we compared CG volumes from 58 children and adults affected by 22q11DS with 64 healthy age- and gender-matched controls. After covarying for total cranium grey matter and age, a bilateral reduced CG grey matter volume, driven by a decrease in anterior CG cortex, was observed among 22q11DS patients. Further post hoc analyses suggest correlations between right CG cortical reductions, low-executive functioning and the occurrence of psychotic symptoms. The CG structural abnormalities observed in 22q11DS are consistent with previous reports in schizophrenic patients and are associated with pre-morbid cognitive impairments. The mechanisms by which these changes may modulate executive functioning and the expression of psychosis are discussed.
\end{abstract}

(C) 2008 Elsevier Ltd. All rights reserved.

\section{Introduction}

Recent studies suggest that individuals at high-risk for psychosis demonstrate structural abnormalities in the cingulate gyrus (CG) (Pantelis et al., 2003), especially reduced grey matter in the anterior cingulate (Borgwardt et al., 2007; Yamasue et al., 2004). As part of the limbic system, the CG is involved in executive function and shares numerous connections with prefrontal cortex and hippocampus (Bush, Luu, \& Posner, 2000), two other regions significantly altered in schizophrenia (Gur, Keshavan, \& Lawrie, 2007; Suzuki et al., 2005). Moreover, cognitive impairments linked with both of these structures, specifically executive function and working memory, are considered as putative endophenotypes and core features for schizophrenia (Bilder et al., 2000; Mohamed, Paulsen, O'Leary, Arndt, \& Andreasen, 1999; Silver, Feldman, Bilker, \& Gur,

\footnotetext{
* Corresponding author. Tel.: +41 2238896 72; fax: +41 223889683. E-mail addresses: federico.dufour@etat.ge.ch, fede.dufour@bluewin.ch (F. Dufour).
}

2003; Snitz, Angus, MacDonald, \& Carter, 2006). Increasing interest is given to identifying such potential endophenotypes, which represent important markers for the complex relationships between genes, brain and related cognitive functions.

It is now established that almost a third of individuals affected by 22q11.2 deletion syndrome (22q11DS), a neurogenetic autosomal dominant condition occurring in approximately 1 in 4000 live births (Oskarsdóttir, Vujic, \& Fasth, 2004), eventually develop schizophrenia (Murphy, Jones, \& Owen, 1999). Moreover, neuropsychological deficits associated with schizophrenia are already apparent in youngsters with 22q11DS. These deficits include impairments in executive function, sustained attention and verbal skills (Lewandowski, Shashi, Berry, \& Kwapil, 2007). Studies on schizotypal manifestations in 22q11DS show that half of the adolescents with the syndrome experience transient positive psychotic symptoms, such as hallucinations and delusions (Baker \& Skuse, 2005; Debbané, Glaser, David, Feinstein, \& Eliez, 2006). Auditory hallucinations represent the earliest symptomatic manifestation of psychosis in children with 22q11DS, which can be observed as early as the age of 9 (Debbané et al., 2006a), and represent a powerful 
predictor for subsequent development of psychosis (Gothelf, Feinstein et al. 2007; Poulton et al., 2000). These observations lend support for the view of psychosis as a continuum (van Os \& Tamminga, 2007), according to which cognitive and clinical manifestations of schizophrenia can be observed, at reduced levels of expression, in individuals prone to psychosis (Brewer et al., 2006).

As previously mentioned, subjects at high-risk for psychosis display brain morphological changes in addition to cognitive changes compared to healthy individuals. Neuroimaging studies of 22q11DS describe how cerebral alterations in the syndrome relate to schizophrenia (Chow, Zipursky, Mikulis, \& Bassett, 2002; Zinkstok \& van Amelsvoort, 2005). Individuals with 22q11DS display general structural brain abnormalities, including reduced total brain tissue, grey and white matter volumes (Eliez, Schmitt, White, \& Reiss, 2000; Kates et al., 2001), increased ventricular and basal ganglia volumes (Eliez, Barnea-Goraly, Schmitt, Liu, \& Reiss, 2002), decreased thalamic, hippocampal as well amygdala volumes (Bish, Nguyen, Ding, Ferrante, \& Simon, 2004; Debbané, Schaer, Farhoumand, Glaser, \& Eliez, 2006; Deboer, Wu, Lee, \& Simon, 2007), and a reduction in cingulate grey matter density (Simon et al., 2005). In schizophrenic 22q11DS subjects compared to nonschizophrenic, further anatomical differences include decreased whole-brain total volume and total white matter and increased total and sulcal cerebrospinal fluid volume (van Amelsvoort et al., 2004). These results provide evidence for a specific pattern of schizophrenic-like cerebral alterations in 22q11DS. Additionally, the executive function deficits in 22q11DS (Lewandowski et al., 2007) have been closely related to structural abnormalities in the anterior CG in schizophrenia (Carter, MacDonald, Ross, \& Stenger, 2001; Morey et al., 2005; Szeszko et al., 2000).

Research has demonstrated that structural cerebral alterations may disrupt related cognitive function (Bush et al., 2000; Karmiloff-Smith et al., 1998), potentially sustaining resulting psychopathological manifestations such as hallucinations (Aleman \& Larøi, 2008). Frith, Friston, Liddle, \& Frackowiak (1992) suggests that the anterior CG is key to positive symptom activity, and recent research supports this claim (Allen, Larøi, McGuire, \& Aleman, 2008). In the verbal self-monitoring hypothesis proposed by Frith et al. (1992), positive symptoms involve misattributing the origin of self-generated mental events (thoughts, intentions, internal speech) to a source other than the self. These self-monitoring deficits, shown to involve the anterior CG (Allen et al., 2007), can promote the expression of hallucinations (Aleman \& Larøi, 2008). Accordingly, both structural and functional alterations in the anterior CG are present among psychotic patients with positive symptoms (Choi et al., 2005; Shergill, Brammer, Williams, Murray, \& McGuire, 2000; Wang et al., 2007). Therefore, given that 22q11DS patients are particularly prone to experience positive symptoms like hallucinations from a young age (Baker \& Skuse, 2005; Debbané, Glaser et al., 2006), a careful analysis of CG structure and associated clinical symptoms seems worthwhile.

The aim of this study is to examine CG structure and its potential relationships with executive dysfunction and positive psychotic symptomatology in a sample of individuals with 22q11DS.

To accurately measure CG morphology, we employed a ROIbased analysis method for its high sensitivity and specificity, rather than voxel-based morphometry (VBM)-analysis, which can sometimes produce artifactual results (Eckert et al., 2006). We conducted this research on a large sample of affected children, adults and healthy controls. As suggested by previous VBM results (Simon et al., 2005), we expected CG volumes to be reduced in 22q11DS subjects. Following previous reports on executive dysfunction and CG alterations in schizophrenic patients (Carter et al., 2001; Morey et al., 2005; Szeszko et al., 2000), we explored whether altered CG morphology is associated with the deficits in executive function frequently observed in 22q11DS (Lewandowski et al., 2007). Finally, given evidence for an implication of CG integrity in the expression of positive psychotic symptoms, we expected to find structural differences in CG volume between psychotic and non-psychotic 22q11DS individuals.

\section{Materials and methods}

\subsection{Subjects}

\subsubsection{2q11DS group}

Fifty-eight patients with 22q11DS aged $6-37$ years ( mean $=15.52 \pm 8.75$ ) participated in the study. Detailed demographic characteristics are presented in Table 1. The sample had a mean full-scale IQ score of $69.03 \pm 11.79$ as measured by the Wechsler Intelligence Scales for Children or Adults (WISC-III and WAIS-III) (Wechsler, 1991, 1997). The 22q11.2 deletion was confirmed in all patients using PCR direct sequencing. Written informed consent was received from all participating subjects, as well as the parents of subjects younger than 18 years of age, in accordance with protocols approved by the Institutional Review Board of Geneva University School of Medicine. At time of participation, a total of 10 patients were taking psychotropic medication, five of which had a diagnosis of schizophrenia.

The presence of positive psychotic symptoms was determined through semistructured interviews with participants affected by 22q11DS and their parents. The parents of participants younger than 18 years responded to a computerized DICA$\mathrm{P}$ (Reich, 2000), administered by a child and adolescent psychiatrist (S.E.). DICA-P software generated DSM-IV diagnoses as well as a listing by diagnostic criteria of all symptoms reported as present or absent. The DICA-P was supplemented with the K-SADS-PL (Kaufman et al., 1997) for evidence of psychosis and mood cycling. Participants older than 18 years were interviewed separately from their parents by the same psychiatrist (S.E.) using the SCID-I to generate DSM-IV diagnoses and criteria (First et al., 1993). This procedure was supplemented with the SADS-PL. The "degree of psychosis" scale (Table 1) represents a description of patients' psychotic symptoms and the severity. This scale has been used in a previous publication (Debbané, Glaser, \& Eliez, 2008).

\subsubsection{2q11DS subgroups}

Psychotic ( $n=24,11$ males and 13 females) and non-psychotic ( $n=18,7$ males and 11 females) subgroups were created from the 22q11DS group for post hoc analyses. This division corresponds to a degree of psychosis $>0$ (psychotic) or $=0$ (non-psychotic). Only patients older than age 9 were used $(n=42)$, given the age at which psychotic symptoms become relevant in the clinical picture of children with 22q11DS (Debbané, Glaser et al., 2006).

These patients also were divided into high-executive functioning ( $n=20,12$ males and 8 females) and low-executive functioning ( $n=20,5$ male and 15 female) subgroups. A composite score (WISC III-Digit span subtest+Stroop interference score) was used to assess level of executive function. Only for the executive function analyses, two of the 42 subjects were excluded due to an absence of data. Table 3 shows detailed group characteristics and Section 2.3 describes the executive function composite score.

\subsubsection{Control group}

The comparison group consisted of 64 healthy individuals aged 6-39 years ( mean $=15.02 \pm 8.09$ ) with a mean IQ of $111.89 \pm 13.02$. An absence of past or present neurological and psychiatric disorders was established during a medical intake interview and by using scores from standardized screening forms (Medical and Developmental History Form, the CBCL for individuals younger than 18, and the SCL-90 for those older than 18).

\subsection{Brain imaging}

MRI was performed on a Philips Intera $1.5 \mathrm{~T}$ scanner; 124 contiguous coronal slices with a thickness of $1.5 \mathrm{~mm}$ and in-plane resolution of $0.94 \mathrm{~mm} \times 0.94 \mathrm{~mm}$ $(\mathrm{TR}=35 \mathrm{~ms}, \mathrm{TE}=6 \mathrm{~ms})$ were acquired. Image optimization was performed in BrainImage 5.2 following standard procedures whose details have been published elsewhere (Reiss et al., 1998; Schaer et al., 2006).

Manual circumscription of the cingulate gyrus ROI was performed based on a previously published protocol (Woodward et al., 2006) developed by the principal investigator (S.E.). Briefly, we first traced left and right CG on sagittal slices $5 \mathrm{~mm}$ lateral to the midline. Sagittal landmarks were used to draw CG boundaries on coronal slices. The CG was delimited medially by the inter-hemispheric cortical surface, and laterally by a line between the deepest extension of the CG sulcus and the deepest extension of CG grey matter adjacent to the corpus callosum (CC), and by the CG sulcus superiorly and the CC or the calcarine fissure inferiorly. A dynamic Talaraich grid (Talaraich \& Tournoux, 1988) was then used to define four sub-regions of the CG: ventral anterior (VA; corresponding to $\mathrm{A} / \mathrm{B} / \mathrm{C}$ boxes of Talairach), dorsal anterior (DA; D/E1 boxes), cingulate body (CinB; E2/E3 boxes) and splenium cingulate (SCin; F/G boxes) (Fig. 1). 
Table 1

Demographic and medical data

\begin{tabular}{|c|c|c|c|c|c|c|c|c|}
\hline & \multicolumn{3}{|c|}{ 22q11DS } & \multicolumn{3}{|c|}{ Controls } & \multicolumn{2}{|l|}{ ANOVA } \\
\hline & $n$ & Mean & S.D. & $n$ & Mean & S.D. & $F$ & $p$ \\
\hline Age & 58 & 15.521 & \pm 8.751 & 64 & 15.024 & \pm 8.099 & 0.106 & 0.745 \\
\hline IQ & 58 & 69.034 & \pm 11.799 & 64 & 111.89 & \pm 13.023 & 360.102 & 0.001 \\
\hline Gender $1 / 2$ & & 1.569 & \pm 0.499 & & 1.609 & \pm 0.491 & 0.202 & 0.654 \\
\hline Male $=1$ & 25 & & & 25 & & & & \\
\hline Female $=2$ & 33 & & & 39 & & & & \\
\hline Degree of psychosis ${ }^{\mathrm{a}}$ & 58 & 1.21 & \pm 1.67 & NA & NA & & & \\
\hline Psychotropic medication & 10 & & 0 & & & & & \\
\hline Schizophrenia & 5 & & 0 & & & & & \\
\hline
\end{tabular}

a Degree of psychosis: 0 = no symptoms lifetime; 1 = hallucination or delusion ( $<3$ lifetime); 2 = hallucination or delusion ( $>3$ lifetime); $3=$ hallucination or delusion ( $m$ onthly basis); 4 = hallucination or delusion (weekly basis); 5 = DSM-IV schizophrenia diagnosis.

For all procedures, two independent raters, blind to the participants' diagnoses (FD, MS), traced the CG volumes of 10 randomly chosen subjects. Intra-class correlation coefficients for total left and right cingulate tissue volumes were 0.94 , indicating good inter-rater reliability for measurements.

\subsection{Statistics}

An alpha of 0.05 (two-tailed) was used as the threshold for statistical significance. Specific covariates including total grey or white matter volumes, age, IQ or psychosis degree were used when necessary to exclude any non-significant freestanding results.

\subsubsection{Volumetric comparisons between 22q11DS and control groups}

First, ANOVA were used to compare total brain tissue, grey and white matter volumes between groups. Second, we used MANCOVA, with total cranium grey or white matter volumes respectively as covariates, to compare CG grey and white matter volumes bilaterally, and then to compare grey matter volumes from the four CG sub-regions for both hemispheres. Significant regional differences were then retested adding age as a covariate.

\subsubsection{Post hoc volumetric analyses within 22q11DS and control subgroups}

For the aforementioned reasons, only patients 9 years of age and older were included in post hoc analyses.

First, we defined high- and low-executive functioning subgroups by averaging the $z$-scores converted from standard scores obtained from the Digit Span and Stroop interference tasks. These tests were specifically chosen given that the anterior CG cognitive division is important for executive control (Bush et al., 2000). We then divided our sample of 22q11DS subjects by high $(z$-score $>0)$ and low $(z$-score $<0)$ executive-functioning individuals. An ANCOVA using age, IQ and degree of psychosis

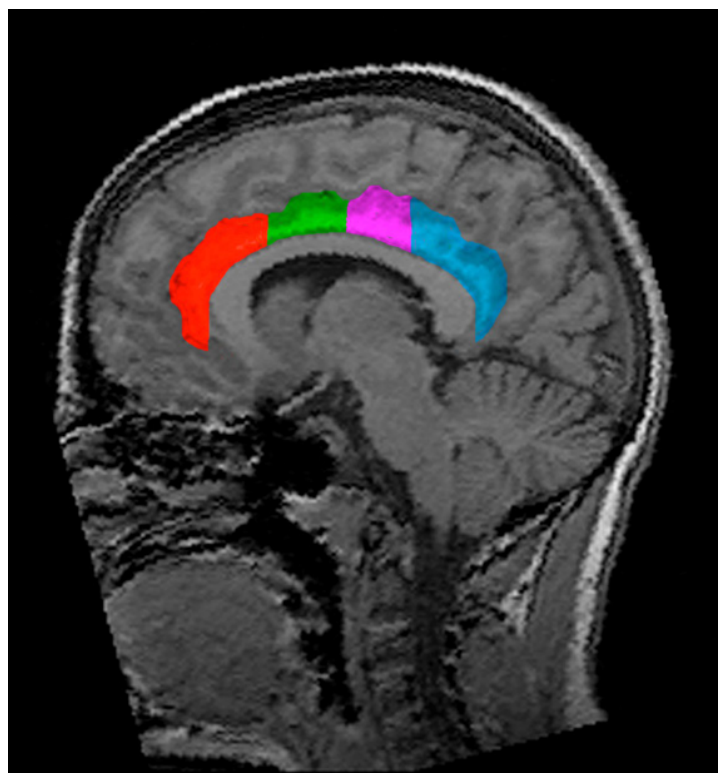

Fig. 1. Sub-regions of the cingulate gyrus are shown: ventral anterior (red), dorsal anterior (green), cingulate body (pink), splenium cingulate (blue). The ROI excluded sub-genual cingulate gyrus. as covariates, and executive function as a group factor was then performed to compare high and low executive functioning subgroups on left/right CG grey matter volumes.

Second, we employed ANCOVA, with age as a covariate and presence of psychotic symptoms as a group factor to test the effect of psychosis on left and right CG grey matter volumes.

Finally, we repeated the same procedure for posterior CG regions (splenium cingulate sub-region), which were not expected to be related to executive function or psychotic symptoms.

We subsequently tested for any significant relationships between executive functioning and CG grey matter volumes within control subjects older than 9 (48 individuals split in 27 high- and 21 low-executive functioning control subjects).

2.3.3. Relationship between executive function and psychotic symptoms in 22q11DS

ANOVA with psychotic symptoms as group factor and the executive function composite mean $z$-score as the dependent variable was used to test a potential relationship between level of executive-functioning and presence of psychotic symptoms.

\section{Results}

\subsection{Volumetric comparisons between 22q11DS subjects and healthy controls}

Subjects with 22q11DS showed a significant reduction in total brain tissue and grey and white matter volumes compared to the control group (total brain tissue: $p=0.001$; total cranium grey matter: $p=0.000$; total cranium white matter: $p=0.001$ ) (Table 2). MANCOVA indicated smaller bilateral CG grey matter volumes in 22q11DS compared to controls (Wilks Lambda: $p=0.002$, left: $p=0.025$; right: $p=0.003$ ). Further delineation of the CG subregions (Fig. 1) revealed that dorsal anterior and cingulate body grey matter volumes were also reduced bilaterally (Wilks Lambda: $p=0.013 ; p=0.004$ for left DA; $p=0.002$ for right DA; $p=0.027$ for left CinB; $p=0.004$ for right CinB). Neither left nor right CG white matter volumes significantly differed between groups. Adding age as a covariate, we observed the same pattern of reduced CG volumes across 22q11DS subjects.

\subsection{Post hoc volumetric analyses within 22q11DS and control subgroups}

A significant reduction in right CG grey matter volume was observed in the low-executive functioning 22q11DS subgroup $(p=0.02)$ compared to the high-executive functioning 22q11DS subgroup. When comparing CG grey matter volumes and executive functioning within control subgroups, ANCOVA did not show any significant differences between groups $(p=0.142)$.

Further, a reduction in right CG grey matter in the 22q11DS psychotic group compared to the 22q11DS non-psychotic group, as well as a trend for reductions in the right DA $(p=0.074)$ and CinB $(p=0.054)$ anterior sub-regions were observed (Table 3$)$. 
Table 2

Volumetric comparisons between 22q11DS subjects and healthy controls

\begin{tabular}{|c|c|c|c|c|c|c|}
\hline & \multicolumn{2}{|c|}{ 22q11DS $(n=58)$} & \multicolumn{2}{|c|}{ Controls $(n=64)$} & \multicolumn{2}{|c|}{ ANCOVA } \\
\hline & Mean & S.D. & Mean & S.D. & $F$ & $p$ \\
\hline Total brain tissue ${ }^{a}$ & 1124.523 & \pm 138.71 & 1244.722 & \pm 102.87 & 29.915 & 0.001 \\
\hline Total cranium grey matter ${ }^{\mathrm{a}}$ & 674.682 & \pm 85.813 & 743.839 & \pm 74.432 & 22.714 & 0.001 \\
\hline Total cranium white matter ${ }^{\mathrm{a}}$ & 449.841 & \pm 90.035 & 500.883 & \pm 70.949 & 12.208 & 0.001 \\
\hline \multicolumn{7}{|l|}{ Cingulate gyrus } \\
\hline Total left grey matter & 11.238 & \pm 1.721 & 12.597 & \pm 1.847 & 5.18 & 0.025 \\
\hline Total right grey matter & 12.167 & \pm 2.36 & 14.274 & \pm 2.16 & 9.31 & 0.003 \\
\hline Total left white matter & 6.94 & \pm 1.352 & 7.288 & \pm 1.214 & 0.004 & 0.951 \\
\hline Total right white matter & 6.677 & \pm 1.534 & 7.247 & \pm 1.129 & 0.864 & 0.355 \\
\hline \multicolumn{7}{|l|}{ Ventral anterior grey matter } \\
\hline Left & 2.484 & \pm 0.889 & 2.862 & \pm 1.29 & 2.059 & 0.154 \\
\hline Right & 3.296 & \pm 1.181 & 3.736 & \pm 1.232 & 1.925 & 0.168 \\
\hline \multicolumn{7}{|l|}{ Dorsal anterior grey matter } \\
\hline Left & 1.904 & \pm 0.349 & 2.314 & \pm 0.619 & 8.46 & 0.004 \\
\hline Right & 2.272 & \pm 0.536 & 2.784 & \pm 0.623 & 10.52 & 0.002 \\
\hline \multicolumn{7}{|l|}{ Cingulate body grey matter } \\
\hline Left & 1.692 & \pm 0.321 & 1.957 & \pm 0.346 & 4.995 & 0.027 \\
\hline Right & 1.739 & \pm 0.374 & 2.091 & \pm 0.378 & 8.721 & 0.004 \\
\hline \multicolumn{7}{|l|}{ Splenium cingulate grey matter } \\
\hline Left & 5.156 & \pm 0.942 & 5.461 & \pm 0.945 & 0.003 & 0.957 \\
\hline Right & 4.859 & \pm 1.025 & 5.661 & \pm 1.237 & 2.816 & 0.096 \\
\hline
\end{tabular}

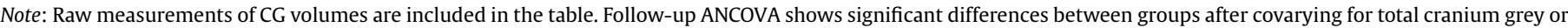
white matter volume. All volumes are expressed in $\mathrm{cm}^{3}$.

a ANOVA was used to statistically compare volumes.

To test whether these results were related to deficits in executive function and psychotic symptoms observed in 22q11DS, we performed the same analyses with the posterior segment of the CG (splenium cingulate sub-region), and did not observe a significant relationship with executive function or psychotic symptoms.

\subsection{Relationship between executive function and psychotic symptoms in 22q11DS}

ANOVA with psychotic symptoms as a group factor and the executive function mean $z$-score as a dependent variable indicated a trend $(p=0.064)$ toward a relationship between low executive functioning and the presence of psychotic symptoms. Indeed, the general distribution of psychotic symptoms among the low- and high-executive functioning 22q11DS individuals shows that $70 \%$ of the low-executive functioning subjects demonstrated psychotic symptoms versus $40 \%$ in the high-functioning subgroup (Table 3 ).

\section{Discussion}

To our knowledge, this is the first investigation of the cingulate gyrus structure using ROI-based analyses in 22q11DS individuals. The results demonstrate bilateral reductions in CG cortical volume compared to normal controls, driven by a decrease in anterior CG grey matter volumes, which remain significant after covarying for age and total grey matter volume. Further, post hoc analyses illustrated a reduction in the right CG grey matter volume in lowexecutive functioning patients, associating right CG alterations in 22q1DS with executive function deficits. We also observed an overall right CG grey matter reduction in the participants with 22q11DS reporting psychotic symptoms, and post hoc analyses revealed a trend toward right anterior CG grey matter reduction in relation to the presence of psychotic symptoms. Decreased statistical power in our post hoc analyses may have prevented the identification of specific CG sub-regional alterations linked to psychosis in 22q11DS. Finally, we observed a trend toward a correlation between lowexecutive functioning and the presence of psychotic symptoms.
Reductions in anterior cingulate grey matter volumes confirm CG alterations in 22q11DS compared to healthy controls, which were first reported by Simon and colleagues (2005) using voxelbased morphometry analyses. These findings are also compatible with anterior CG structural abnormalities found in individuals at high-risk for psychosis (Borgwardt et al., 2007), as well as in schizophrenia (Baiano et al., 2007). Using support from the literature on psychosis, in this discussion we will focus on the following points: (1) the implication of a relationship between executive function deficits and anterior CG changes; (2) the potential involvement of the anterior CG in the expression of psychotic symptoms in 22q11DS; and (3) suggestions for future explorations of brain structure and cognitive functions leading to positive symptom expression.

Cognitive studies have shown that executive function and working memory deficits related to the anterior CG (Carter et al., 2001; Morey et al., 2005; Szeszko et al., 2000) are present in most schizophrenic individuals (Bilder et al., 2000; Mohamed et al., 1999; Silver et al., 2003; Snitz et al., 2006). Neuroimaging studies have directly linked these deficits to the CG. Indeed, executive and working memory tasks normally activate the caudal part of the anterior CG (Bush et al., 2000), and a significant positive correlation between the volume of the right anterior CG and the ability to perform a go/no-go task has been previously reported (Bush et al., 2000). Thus, the relationship between right CG cortical reductions and low-executive functioning in 22q11DS patients may represent an endophenotypic marker signaling neurocognitive deficits associated with schizophrenia. A recent study of children and adolescents with 22q11DS reporting "schizophrenic-like" executive functioning deficits (Lewandowski et al., 2007) further supports this idea.

Pronounced CG structural alterations in individuals with psychosis and 22q11DS may provoke functional disruptions in a cerebral network responsible for the development of positive symptoms such hallucinations. The existing literature on high-risk and schizophrenic samples implicates the CG in the pathology of psychosis (Borgwardt et al., 2007; Pantelis et al., 2003; Yamasue et al., 2004). Suzuki and colleagues (2005) suggest that loss of 
Table 3

Post hoc analyses among 22q11DS subjects aged above 9

\begin{tabular}{|c|c|c|c|c|c|c|c|}
\hline & \multicolumn{3}{|c|}{ Psychotic 22q11DS subgroup ( $n=24)$} & \multicolumn{2}{|c|}{ Non-psychotic 22q11DS subgroup ( $n=18$ ) } & \multicolumn{2}{|c|}{ ANCOVA } \\
\hline & Mean & S.D. & & Mean & S.D. & $F$ & $p$ \\
\hline \multicolumn{8}{|c|}{ Psychosis versus non-psychosis volumetric comparisons $(n=42)$} \\
\hline Age $^{a}$ & 20.482 & \pm 7.8 & & 16.255 & \pm 8.561 & 2.763 & 0.104 \\
\hline IQ & 64.208 & \pm 10.6 & & 72.111 & \pm 10.867 & 5.555 & 0.066 \\
\hline \multicolumn{8}{|l|}{ Cingulate gyrus } \\
\hline Total left grey matter & 10.919 & \pm 1.7 & & 11.661 & \pm 2.083 & 0.733 & 0.397 \\
\hline Total right grey matter & 11.438 & \pm 2.5 & & 13.485 & \pm 2.22 & 4.479 & 0.041 \\
\hline \multicolumn{8}{|c|}{ Dorsal anterior grey matter } \\
\hline Left & 1.852 & \pm 0.3 & & 1.908 & \pm 0.345 & 0.13 & 0.72 \\
\hline Right & 2.111 & \pm 0.5 & & 2.519 & \pm 0.541 & 3.371 & 0.074 \\
\hline \multicolumn{8}{|c|}{ Cingulate body grey matter } \\
\hline Left & 1.58 & \pm 0.2 & & 1.748 & \pm 0.348 & 1.417 & 0.241 \\
\hline \multirow[t]{3}{*}{ Right } & 1.602 & \pm 0.2 & & 1.919 & \pm 0.483 & 3.937 & 0.054 \\
\hline & \multicolumn{3}{|c|}{ High 22q11DS subgroup $(n=20)$} & \multicolumn{2}{|c|}{ Low 22q11DS subgroup $(n=20)$} & \multicolumn{2}{|l|}{ ANCOVA } \\
\hline & Mean & & & Mean & S.D. & $F$ & $p$ \\
\hline \multicolumn{8}{|c|}{ High versus low executive functioning volumetric comparisons $(n=40)$} \\
\hline Age $^{\mathrm{a}}$ & 15.817 & & & 20.062 & \pm 8.229 & 3.12 & 0.085 \\
\hline $\mathrm{IQ}^{\mathrm{a}}$ & 72.2 & & & 64.85 & \pm 11.065 & 5.171 & 0.029 \\
\hline \multicolumn{8}{|l|}{ Cingulate gyrus } \\
\hline Total left grey matter & 11.75 & & & 10.843 & \pm 1.735 & 1.293 & 0.324 \\
\hline Total right grey matter & 13.615 & & & 11.342 & \pm 2.859 & 6.142 & 0.020 \\
\hline \multicolumn{8}{|c|}{ Dorsal anterior grey matter } \\
\hline Left & 1.915 & & & 1.838 & \pm 0.291 & 0.279 & 0.730 \\
\hline Right & 2.506 & & & 2.136 & \pm 0.631 & 2.45 & 0.105 \\
\hline \multicolumn{8}{|c|}{ Cingulate body grey matter } \\
\hline Left & 1.756 & & & 1.556 & \pm 0.255 & 2.262 & 0.339 \\
\hline Right & 1.918 & & & 1.593 & \pm 0.362 & 3.576 & 0.117 \\
\hline \multicolumn{8}{|c|}{ Executive functioning-psychotic symptoms relationship } \\
\hline Psychotic subjects & $n=8$ & & & \multirow{2}{*}{\multicolumn{4}{|c|}{$\begin{array}{r}n=14 \\
n=6\end{array}$}} \\
\hline \multirow[t]{3}{*}{ Non-psychotic subjects } & $n=12$ & & & & & & \\
\hline & & \multicolumn{2}{|c|}{ Psychotic subjects $(n=22)$} & \multicolumn{2}{|c|}{ Non-psychotic subjects $(n=18)$} & \multicolumn{2}{|c|}{ ANCOVA } \\
\hline & & Mean & S.D. & & S.D. & $F$ & $p$ \\
\hline Executive function composi & score $^{a}$ & -0.217 & \pm 0.637 & & \pm 0.789 & 3.641 & 0.064 \\
\hline
\end{tabular}

Note: Raw measurements of CG volumes are included in table. Follow-up ANCOVA shows significant differences between groups. All volumes are expressed in cm ${ }^{3}$.

a ANOVA was used to statistically compare groups.

inhibitory control, typically regulated in networks involving the prefrontal cortex and the anterior CG (Kerns et al., 2004), may be significant to the development of such symptoms, related to an anterior CG grey matter volume reduction in schizophrenia (Choi et al., 2005; Wang et al., 2007). Concordantly, Allen et al. (2008) review several reports illustrating anterior CG activity deficits during hallucinatory experiences. For example, the authors suggest that abnormal anterior CG and temporal cortex activation leads, in patients with auditory verbal hallucinations, to the misattribution of inner speech to an external source (Allen et al., 2007). Moreover, abnormal connections between the temporal and anterior CG cortex also contribute to verbal self-monitoring deficits, further sustaining auditory verbal hallucinations (Johns \& McGuire, 1999; Mechelli et al., 2007). Finally, consistent with our structural findings of an altered right CG volume in 22q11DS patients with psychotic symptoms, Shergill et al. (2000) report the involvement of a large network of cortical areas prominently in the right hemisphere, including CG, in auditory hallucinations.

Although our data point to an association between right CG alteration, executive function and psychotic expression, it is difficult at this point to differentiate between the cause and effect of their putative contributions. Considering that CG grey matter reduction is a common finding among 22q11DS people compared to healthy individuals, two developmental hypotheses may be likely: (1) CG cortical alterations may disturb executive functioning in 22q11DS patients, thereby increasing the risk for psychotic symptom expression, or (2) CG cortical alterations may directly support hallucination-proneness thereby affecting executive function deficits in 22q11DS subjects. To date, longitudinal studies in 22q11DS find that cerebral alterations, most notably in dorsolateral prefrontal cortex, are related to cognitive alterations (verbal IQ decline) that accompany the rise of psychotic symptom expression (Debbané, Glaser et al., 2006; Gothelf et al., 2005). However, a direct relationship between developmental brain abnormalities and psychosis expression in 22q11DS has yet to be found (Gothelf, Penniman, Gu, Eliez, \& Reiss, 2007).

One limitation of our study is that we cannot exclude an effect of IQ concerning the structural results between 22q11DS subjects and the control group. Indeed, the groups show significant differences in IQ, which are likely correlated with brain grey matter volume (Reiss, Abrams, Singer, Ross, \& Denckla, 1996). Low IQ is probably a general result of the many specific developmental factors interacting in 22q11DS, like the ones specifically tested in this study. Covarying for IQ is an ongoing debate in research on neurodevelopmental syndromes because it often means covarying out the very effects in question. However, within-group analyses 
clearly show the association between right CG alteration and psychotic symptoms in 22q11DS, independent of age or IQ. Another limitation is our inability to test for any effects of medication between the 10 individuals with 22q11DS following pharmacological treatment and healthy subjects because of the large variety of psychotropic drugs (neuroleptic, anti-epileptic, benzodiazepine, methylphenidate) prescribed to the participants. Finally, the assessment of psychotic symptoms will necessitate finer evaluation to better understand their developmental process in 22q11DS. Future studies employing dimensional measures characterizing frequency and intensity of hallucinations and delusions, and the distress and perturbation caused by these symptoms, may help to clarify the complex interactions between brain morphology, cognitive profile and the unfolding of positive symptoms psychosis.

\section{Acknowledgments}

This work was supported by Swiss National Research Funds to SE (PP00B-102864) and MS (323500-111165), in addition to a grant from the NARSAD Institute to SE. We thank our collaborators at the Center for Biomedical Imaging (CIBM), especially J. Delavelle, F. Henry and F. Lazeyras for their support and assistance with data collection, as well the EPFL, and the Leenaards and Louis Jeantet Foundations. We would also like to acknowledge S. Dahoun and S. Antonarakis for their ongoing collaboration in the Department of Genetics. This work is made possible by the Swiss and French deletion22q11 parent associations, Connect22 and Generation 22.

Financial disclosures: All authors of this manuscript have no biomedical financial support or conflict of interests.

\section{References}

Aleman, \& Larøi. (2008). Hallucinations: The science of idiosyncratic perception. American Psychological Association.

Allen, P., Amaro, E., Fu, C. H., Williams, S. C., Brammer, M. J., Johns, L. C., \& McGuire, P. K. (2007). Neural correlates of the misattribution of speech in schizophrenia. British Journal of Psychiatry, 190, 162-169.

Allen, P., Larøi, F., McGuire, P. K., \& Aleman, A. (2008). The hallucinating brain: A review of structural and functional neuroimaging studies of hallucinations. Neuroscience and Biobehavioral Reviews, 32, 175-191.

Baiano, M., David, A., Versace, A., Churchill, R., Balestrieri, M., \& Brambilla, P. (2007). Anterior cingulate volumes in schizophrenia: A systematic review and a metaanalysis of MRI studies. Schizophrenia Research, 93, 1-12.

Baker, K. D., \& Skuse, D. H. (2005). Adolescents and young adults with 22q11 deletion syndrome: Psychopathology in an at-risk group. British Journal of Psychiatry, 186 115-120.

Bilder, R. M., Goldman, R. S., Robinson, D., Reiter, G., Bell, L., \& Bates, J. A. (2000). Neuropsychology of first-episode schizophrenia: Initial characterization and clinical correlates. American Journal of Psychiatry, 157, 549-559.

Bish, J. P., Nguyen, V., Ding, L., Ferrante, S., \& Simon, T. J. (2004). Thalamic reductions in children with chromosome 22q11.2 deletion syndrome. Neuroreport, 15 , 1413-1415.

Borgwardt, S. J., Riecher-Rössler, A., Dazzan, P., Chitnis, X., Aston, J., Drewe, M., et al. (2007). Regional grey matter volume abnormalities in the at risk mental state. Biological Psychiatry, 61, 1148-1156.

Brewer, W. J., Wood, S. J., Phillips, L. J., Francey, S. M., Pantelis, C., Yung, A. R., et al. (2006). Generalized and specific cognitive performance in clinical high-risk cohorts: A review highlighting potential vulnerability markers for psychosis. Schizophrenia Bulletin, 32, 538-555.

Bush, G., Luu, P., \& Posner, M. I. (2000). Cognitive and emotional influences in anterior cingulate cortex. Trends in Cognitive Sciences, 4, 215-222.

Campbell, L. E., Daly, E., Toal, F., Stevens, A., Azuma, R., Catani, M., et al. (2006). Brain and behaviour in children with 22q11.2 deletion syndrome: A volumetric and voxel-based morphometry MRI study. Brain, 129, 1218-1228.

Carter, C. S., MacDonald, A. W., III, Ross, L. L., \& Stenger, V. A. (2001). Anterior cingulate cortex activity and impaired self-monitoring of performance in patients with schizophrenia: An event-related fMRI study. American Journal of Psychiatry, 158, 1423-1428.

Choi, J. S., Kang, D. H., Kim, J. J., Ha, T. H., Roh, K. S., Youn, T., \& Kwon, J. S. (2005). Decreased caudal anterior cingulate gyrus volume and positive symptoms in schizophrenia. Psychiatry Research, 139, 39-47.
Chow, E. W., Zipursky, R. B., Mikulis, D. J., \& Bassett, A. S. (2002). Structural brain abnormalities in patients with schizophrenia and 22q11 deletion syndrome. Biological Psychiatry, 51, 208-215.

Debbané, M., Glaser, B., David, M. K., Feinstein, C., \& Eliez, S. (2006). Psychotic symptoms in children and adolescents with 22q11.2 deletion syndrome: Neuropsychological and behavioral implications. Schizophrenia Research, 84, 187-193.

Debbané, M., Schaer, M., Farhoumand, R., Glaser, B., \& Eliez, S. (2006). Hippocampal volume reduction in 22q11.2 deletion syndrome. Neuropsychologia, 44, 2360-2365

Debbané, M., Glaser, B., \& Eliez, S. (2008). Encoding and retrieval processes in velocardio-facial syndrome (VCFS). Neuropsychology, 22, 226-234.

Deboer, T., Wu, Z., Lee, A., \& Simon, T. J. (2007). Hippocampal volume reduction in children with chromosome 22q11.2 deletion syndrome is associated with cognitive impairment. Behavioral Brain Function, 3, 54.

Eckert, M. A., Tenforde, A., Galaburda, A. M., Bellugi, U., Korenberg, J. R., Mills, D., \& Reiss, A. L. (2006). To modulate or not to modulate: Differing results in uniquely shaped Williams syndrome brains. Neuroimage, 32, 1001-1007.

Eliez, S., Barnea-Goraly, N., Schmitt, J. E., Liu, Y., \& Reiss, A. L. (2002). Increased basal ganglia volumes in velo-cardio-facial syndrome (deletion 22q11.2). Biological Psychiatry, 52, 68-70.

Eliez, S., Schmitt, J. E., White, C. D., \& Reiss, A. L. (2000). Children and adolescents with velocardiofacial syndrome: A volumetric MRI study. American Journal of Psychiatry, 157, 409-415.

First, M. B., Opler, L. A., Hamilton, R. M., Linder, J., Linfield, L. S., Silver, J. M., et al. (1993). Evaluation in an inpatient setting of DTREE, a computerassisted diagnostic assessment procedure. Comprehensive Psychiatry, 34, 171175.

Frith, C. D., Friston, K. J., Liddle, P. F., \& Frackowiak, R. S. (1992). PET imaging and cognition in schizophrenia. Journal of the Royal Society of Medicine, 85, 222-224.

Gothelf, D., Eliez, S., Thompson, T., Hinard, C., Penniman, L., Feinstein, C., et al. (2005). COMT genotype predicts longitudinal cognitive decline and psychosis in $22 \mathrm{q} 11.2$ deletion syndrome. Nature Neuroscience, 8, 1500-1502.

Gothelf, D., Feinstein, C., Thompson, T., Gu, E., Penniman, L., Van Stone, E., et al. (2007). Risk factors for the emergence of psychotic disorders in adolescents with 22q11.2 deletion syndrome. American Journal of Psychiatry, 164, 663-669.

Gothelf, D., Penniman, L., Gu, E., Eliez, S., \& Reiss, A. L. (2007). Developmental trajectories of brain structure in adolescents with 22q11.2 deletion syndrome: A longitudinal study. Schizophrenia Research, 96, 72-81.

Gur, R. E., Keshavan, M. S., \& Lawrie, S. M. (2007). Deconstructing psychosis with human brain imaging. Schizophrenia Bulletin, 33, 921-931.

Johns, L. C., \& McGuire, P. K. (1999). Verbal self-monitoring and auditory hallucinations in schizophrenia. Lancet, 353, 469-470.

Karmiloff-Smith, A., Tyler, L. K., Voice, K., Sims, K., Udwin, O., Howlin, P., et al. (1998). Linguistic dissociations in williams syndrome: Evaluating receptive syntax in on-line and off-line tasks. Neuropsychologia, 36, 343-351.

Kates, W. R., Burnette, C. P., Jabs, E. W., Rutberg, J., Murphy, A. M., Grados, M., et al. (2001). Regional cortical white matter reductions in velocardiofacial syndrome: A volumetric MRI analysis. Biological Psychiatry, 49, 677-684.

Kaufman, J., Birmaher, B., Brent, D., Rao, U., Flynn, C., Moreci, P., et al. (1997). Schedule for Affective Disorders and Schizophrenia for School-Age Children-Present and Lifetime Version (K-SADS-PL): Initial reliability and validity data. Journal of the American Academy of Child and Adolescent Psychiatry, 36, 980-988.

Kerns, J. G., Cohen, J. D., MacDonald, A. W., Cho, R. Y., Stenger, V. A., \& Carter, C. S. (2004). Anterior cingulate conflict monitoring and adjustments in control. Science, 303, 1023-1026.

Lewandowski, K. E., Shashi, V., Berry, P. M., \& Kwapil, T. R. (2007). Schizophreniclike neurocognitive deficits in children and adolescents with 22q11 deletion syndrome. American Journal of Medical Genetics. Part B, Neuropsychiatric Genetics, 144, 27-36.

Mechelli, A., Allen, P., Amaro, E., Fu, C. H., Williams, S. C., Brammer, M. J., et al. (2007) Misattribution of speech and impaired connectivity in patients with auditory verbal hallucinations. Human Brain Mapping, 28, 1213-1222.

Mohamed, S., Paulsen, J. S., O'Leary, D., Arndt, S., \& Andreasen, N. (1999). Generalized cognitive deficits in schizophrenia: A study of first-episode patients. Archives of General Psychiatry, 56, 794-854.

Morey, R. A., Inan, S., Mitchell, T. V., Perkins, D. O., Lieberman, J. A., \& Belger, A. (2005). Imaging frontostriatal function in ultra-high-risk, early, and chronic schizophrenia during executive processing. Archives of General Psychiatry, 62, 254-262.

Murphy, K. C., Jones, L. A., \& Owen, M. J. (1999). High rates of schizophrenia in adults with velo-cardio-facial syndrome. Archives of General Psychiatry, 56, 940-945.

Oskarsdóttir, S., Vujic, M., \& Fasth, A. (2004). Incidence and prevalence of the 22q11 deletion syndrome: A population-based study in Western Sweden. Archives of Disease in Childhood, 89, 148-151.

Pantelis, C., Velakoulis, D., McGorry, P. D., Wood, S. J., Suckling, J., Phillips, L. J., et al. (2003). Neuroanatomical abnormalities before and after onset of psychosis: A cross-sectional and longitudinal MRI comparison. Lancet, 361, 281-288.

Poulton, R., Caspi, A., Moffitt, T. E., Cannon, M., Murray, R., \& Harrington, H. (2000) Children's self-reported psychotic symptoms and adult schizophreniform disorder: A 15-year longitudinal study. Archives of General Psychiatry, 57, 1053-1058.

Reich, W. (2000). Diagnostic interview for children and adolescents. Journal of American Academy of Child and Adolescent Psychiatry, 39, 59-66.

Reiss, A. L., Abrams, M. T., Singer, H. S., Ross, J. L., \& Denckla, M. B. (1996). Brain development, gender and IQ in children: A volumetric imaging study. Brain, 119, 1763-1774. 
Reiss, A. L., Hennessey, J. G., Rubin, M., Beach, L., Abrams, M. T., Warsofsky, I. S., et al. (1998). Reliability and validity of an algorithm for fuzzy tissue segmentation of MRI. Journal of Computer Assisted Tomography, 22, 471-479.

Schaer, M., Schmitt, J. E., Glaser, B., Lazeyras, F., Delavelle, J., \& Eliez, S. (2006). Abnormal patterns of cortical gyrification in velo-cardio-facial syndrome (deletion 22q11.2): An MRI study. Psychiatry Research, 146, 1-11.

Shergill, S. S., Brammer, M. J., Williams, S. C., Murray, R. M., \& McGuire, P. K. (2000). Mapping auditory hallucinations in schizophrenia using functional magnetic resonance imaging. Archives of General Psychiatry, 57, 1033-1038.

Silver, H., Feldman, P., Bilker, W., \& Gur, R. C. (2003). Working memory deficit as a core neuropsychological dysfunction in schizophrenia. American Journal of Psychiatry, $160,1809-1816$

Simon, T. J., Ding, L., Bish, J. P., McDonald-McGinn, D. M., Zackai, E. H., \& Gee, J. (2005). Volumetric, connective, and morphologic changes in the brains of children with chromosome 22q11.2 deletion syndrome: An integrative study. Neuroimage, 25, 169-180.

Snitz, B. E., Angus, W., MacDonald, III, \& Carter, C. S. (2006). Cognitive deficits in unaffected first-degree relatives of schizophrenia patients: A meta-analytic review of putative endophenotypes. Schizophrenia Bulletin, 32, 179-194.

Suzuki, M., Zhou, S. Y., Takahashi, T., Hagino, H., Kawasaki, Y., Niu, L., et al. (2005). Differential contributions of prefrontal and temporolimbic pathology to mechanisms of psychosis. Brain, 128, 2109-2122.

Szeszko, P. R., Bilder, R. M., Lencz, T., Ashtari, M., Goldman, R. S., Reiter, G., et al. (2000). Reduced anterior cingulate gyrus volume correlates with executive dysfunction in men with first-episode schizophrenia. Schizophrenia Research, 43, 97-108.
Talaraich, J., \& Tournoux, P. (1988). Co-planar stereotaxic atlas of the human brain. New York: Thieme.

van Amelsvoort, T., Daly, E., Henry, J., Robertson, D., Ng, V., Owen, M., et al. (2004). Brain anatomy in adults with velocardiofacial syndrome with and without schizophrenia: Preliminary results of a structural magnetic resonance imaging study. Archives of General Psychiatry, 61, 1085-1096.

van Os, J., \& Tamminga, C. (2007). Deconstructing psychosis. Schizophrenia Bulletin, 33, 861-862.

Wang, L., Hosakere, M., Trein, J. C., Miller, A., Ratnanather, J. T., Barch, D. M., et al. (2007). Abnormalities of cingulate gyrus neuroanatomy in schizophrenia. Schizophrenia Research, 93, 66-78.

Wechsler, D. (1991). Wechsler intelligence scale for children (3rd edn). The Psychological Corporation.

Wechsler, D. (1997). Wechsler intelligence scale for adults (3rd edn). The Psychological Corporation.

Woodward, S. H., Kaloupek, D. G., Streeter, C. C., Martinez, C., Schaer, M., \& Eliez, S. (2006). Decreased anterior cingulate volume in combat-related PTSD. Biological Psychiatry, 59, 582-587.

Yamasue, H., Iwanami, A., Hirayasu, Y., Yamada, H., Abe, O., Kuroki, N., et al. (2004). Localized volume reduction in prefrontal, temporolimbic, and paralimbic regions in schizophrenia: An MRI parcellation study. Psychiatry Research, 131 195-207.

Zinkstok, J., \& van Amelsvoort, T. (2005). Neuropsychological profile and neuroimaging in patients with 22Q11 2 Deletion Syndrome: A review. Child Neuropsychology, 11, 21-37. 